\title{
The dynamics of copper intercalated molybdenum ditelluride
}

\author{
Nicolas Onofrio ${ }^{1}$, David Guzman ${ }^{2}$ and Alejandro Strachan ${ }^{2 *}$ \\ ${ }^{1}$ Department of Applied Physics, The Hong Kong Polytechnic University, Hong Kong SAR \\ ${ }^{2}$ School of Materials Engineering and Birck Nanotechnology Center Purdue University, West Lafayette, \\ IN 47906 USA
}

\begin{abstract}
Layered transition metal dichalcogenides are emerging as key materials in nanoelectronics and energy applications. Predictive models to understand their growth, thermomechanical properties and interactions with metals are needed in order to accelerate their incorporation into commercial products. Interatomic potentials enable large-scale atomistic simulations at the device level, beyond the range of applications of first principle methods. We present a ReaxFF reactive force field to describe molybdenum ditelluride and its interactions with copper. We optimized the force field parameters to describe the properties of layered $\mathrm{MoTe}_{2}$ in various phases, the intercalation of $\mathrm{Cu}$ atoms and clusters within its van der Waals gap, including a proper description of energetics, charges and mechanical properties. The training set consists of an extensive set of first principle calculations computed from density functional theory. We use the force field to study the adhesion of a single layer $\mathrm{MoTe}_{2}$ on a $\mathrm{Cu}(111)$ surface and the results are in good agreement with density functional theory, even though such structures were not part of the training set. We characterized the mobility of the $\mathrm{Cu}$ ions intercalated into $\mathrm{MoTe}_{2}$ under the presence of an external electric fields via molecular dynamics simulations. The results show a significant increase in drift velocity for electric fields of approximately $0.4 \mathrm{~V} / \AA$ and that mobility increases with $\mathrm{Cu}$ ion concentration.
\end{abstract}

Keywords: molecular dynamics, ReaxFF, $\mathrm{MoTe}_{2}$, intercalation, $\mathrm{TMD} /$ metal interface

\section{Introduction}

The integration of single-layer and few-layers transition metal dichalcogenides (TMDs) into nanoelectronics [1] is paving the way for future developments of low power, ultrathin electronic devices [2, 3].

\footnotetext{
${ }^{*}$ Corresponding author: strachan@purdue.edu
} 
The versatile chemical composition and phase stability of TMDs results in a wide range of electronic properties from metallic to wide band gap semiconductor [4. Additionally, the two dimensional nature of TMDs enables its hybridization and integration with various materials via intercalation [5, 6, doping [7, 6, heterostructure [8, 6] and interfaces [9. Although the majority of efforts so far have been devoted to TMD-based transistors [10, 11, TMDs are rapidly finding other applications including metallic interconnects [12] (see also Cu-graphene hybrids [13]), non-volatile memory devices [14, 15, 16] and electro/mechanical switches have been predicted from theory [17, 18].

An important limitation of TMD-based electronics lies in the poor hybridization between metals and TMDs resulting in high interface resistance 9. Therefore, in addition to the inherent Schottky barrier at the metal/semiconductor interface, a tunneling barrier arise from the van der Waals (vdW) nature of the metal-TMD interaction. Moreover, the vdW interaction between multiple layers of TMDs also limits carrier injection at the interface with the metal contact. Various metals have been investigated in order to improve contacts [19] as well as local TMD processing such as intercalation, phase engineering [20] and chemical doping [10]. Due of the wide compositional flexibility of TMDs and the various metals of interest, brute force experimental optimizations are likely to be ineffective and predictive computational techniques can help narrow down experimental studies to the most promising candidates. Electronic structure calculations using density functional theory (DFT), including corrections for the nonlocal dispersive forces part of vdW interactions [21, 22, have played and continue to play a key role in this field [11, 23, 24, 25]. However, the computational intensity of DFT calculations has limited its application to relatively small size, model systems composed of few hundreds of atoms. Thus, most of the DFT studies have focused on defect free TMD/metal interfaces (limited by the small size of the supercell) and little is known about the nanostructure of the interface.

Direct synthesis of single to few layers TMDs have been demonstrated however, even with sophisticated chemical vapor deposition (CVD) techniques [26, 27, TMDs present high defect density [28]. Understanding defect formation in TMDs would not only enable the synthesis of large area, defect-free layers but would also provide the knowledge for defect-controlled TMD growth. Structural defects in TMDs, including point defects and grain boundary influence its electronic, thermal and mechanical properties [29, 30, 31]. Unfortunately, the atomistic mechanisms of nucleation and growth during the CVD process remain unclear.

Large-scale atomistic simulations would be critical to study the interactions between defects and interfaces and to provide atomistic insight into the process of synthesis and growth of these materials. This paper contributes to bridge the gap between first principle calculations and experiments via the 
development of the first reactive force field capable of describing a TMD, a metal and their interactions, specifically molybdenum ditelluride and $\mathrm{Cu}$. We use the ReaxFF formalism that has shown the flexibility to describe a wide range of materials including metals, semiconductors, their oxides 32, 33 and molecular materials [34, 35, 36]. The force field is parameterized to describe various phases of $\mathrm{Cu}, \mathrm{Mo}$ and $\mathrm{MoTe}_{2}$ as well as the corresponding interfaces, the intercalation of $\mathrm{Cu}$ atoms and small clusters in the vdW gap of multiple layers of $\mathrm{MoTe}_{2}$ including a proper description of the relative energies, charges and mechanical properties.

The rest of the paper is organized as follows. In Section 2 we present details about the simulations and methods. Section 3 describes the optimized force field and presents its comparison with DFT calculations. In Sections 4 and 5 we study the interface between monolayer $\mathrm{MoTe}_{2}$ and a Copper slab and the dynamics of $\mathrm{Cu}$-intercalated $\mathrm{MoTe}_{2}$ under various external voltages, respectively. We finally draw conclusions in Section 6

\section{Force field optimization approach and methods}

ReaxFF is a bond order interatomic potential and naturally allows bond breaking and forming during molecular dynamics (MD) simulations [37. The total energy is defined as the sum of various contributions including covalent (bond stretch, angles, torsions, and over/under coordination) and non-bonded interactions (van der Waals and Coulomb); additional terms to account for lone pair and bond conjugation. Electrostatic interactions are described using environment-dependent partial atomic charges which are computed at each step of the simulation using the charge equilibration method [38, 39] (QEq). All contributions to the covalent energy depend on the partial bond order between atoms, a function that smoothly decays to zero when the bond between atoms is broken. Details on the mathematical form of ReaxFF can be found in the original paper, Ref. 37] and a recent review of applications in Ref. 40. In order to determine the parameters that describe the functional form of the interaction, the force field has to be optimized against a training set consisting of first principle calculations or a combination between first principles calculations and experiments. The parameterization of such a force field is complex due to the large number of parameters and the need for extensive training sets and time-consuming optimization cycles. Thus, despite the enormous progress to date [40, several materials of technological importance lack accurate force field descriptions. 


\subsection{DFT simulation details}

All DFT calculations have been performed with the Vienna Ab initio Simulation Package (VASP) [41, 42. within the generalized gradient approximation as proposed by Perdew, Burke, and Ernzerhof (PBE) [43]. In addition, we use the non-local vdW density functional of Langreth and Lundqvist [44, 45] (vdW-DF) throughout the paper to correct for the London dispersion, poorly described by PBE functionals. Atomic structures have been relaxed until energies and forces reach a tolerance of $1 \times 10^{-4} \mathrm{eV}$ and $1 \times 10^{-2} \mathrm{eV} / \AA$, respectively. The kinetic energy cutoff for the plane-wave basis was set to $500 \mathrm{eV}$ and we integrate the $\mathrm{k}$-mesh with various number of points depending on the size of the supercell. We use a dense $8 \times 8 \times 8$ k-mesh for bulk $\mathrm{Cu}$ and Mo, an intermediate $4 \times 4 \times 2$ grid for bulk $\mathrm{MoTe}_{2}$ and $\mathrm{Cu}$-intercalated $\mathrm{MoTe}_{2}$ and gamma point calculations have been performed on large cells used to compute Cu mobility. We verified that the kinetic energy cutoff chosen provides well converged structures with respect to energy, force and stress. Diffusion of $\mathrm{Cu}$ ions in the vdW gap of $\mathrm{MoTe}_{2}$ and minimum energy path between $\mathrm{H} \rightarrow \mathrm{T}$ ' phases of $\mathrm{MoTe}_{2}$ have been computed using the nudged elastic band 46] (NEB) method, as implemented in VASP. Additionally, we performed $a b$ initio MD simulations at $500 \mathrm{~K}$ with a kinetic energy cutoff of 300 $\mathrm{eV}$ and timestep of $1.5 \mathrm{fs}$ in order to overcome the energy barrier corresponding to the dissociation of a $\mathrm{Cu}_{3}$ cluster intercalated in $\mathrm{MoTe}_{2}$.

\subsection{Parameter optimization}

In order to optimize the force field we use an in-house code written in Python that implements a Monte Carlo-based (MC) simulated annealing algorithm [47] and coupled to the LAMMPS simulator [48] (via its Python interface) to evaluate energies, forces, pressures and charges of molecules and crystals included in the training set. This method has been used previously to optimize force fields for oxides [49] and metals 50. The training set is included as an XML database of DFT calculations describing the dissociation of small molecules and equation of states for crystals. The goal of the simulated annealing optimization is to minimize a total error function $\left(\epsilon_{t o t}\right)$ defined as the sum of individual discrepancies between the force field and DFT data, corresponding to energy (E), force (f), pressure (P) and charge (Q) as:

$$
\epsilon_{\text {tot }}=\sum_{X=E, f, P, Q} \frac{\left(X^{\operatorname{Reax} F F}-X^{D F T}\right)^{2}}{N_{X}^{2}}
$$

with $N_{X}$ a set of normalization constants chosen such that each individual error appears with equivalent weight; these parameters are also used to establish the relative importance between different quan- 
tities in the training set.

Before starting the simulated annealing run we perform an initial sensitivity analysis for each parameter in the force field in order to determine the steps by which each parameter will be stochastically modified during the MC run. A Metropolis criterion allows the random selection of some "non-optimum" parameters in order to expand the exploration space and avoid the force field function becoming trapped in local minima. The temperature used for the Metropolis acceptance is decreased during the simulation in order to converge towards the global minimum. We note that the QEq parameters are optimized first in order to reproduce partial atomic charges of the structures in the training set obtained from Bader analysis [51] of the DFT runs. This is done by setting $\epsilon_{E}, \epsilon_{f}$ and $\epsilon_{P}$ to zero in the energy expression so that only partial charges are compared. The simulated annealing code distributes MD simulations in parralel (using IPython for parallel computing) and at its current stage of development can perform 1,000 single point calculations in approximatively 5 seconds over 64 cores.

\section{$3 \mathrm{Cu}-\mathrm{MoTe}_{2}$ reactive force field}

$\mathrm{MoTe}_{2}$ has recently received significant attention because of the discovery of a metallic T' phase [52, 53 ] energetically close [17, 18, to its ground state semiconducting $\mathrm{H}$ phase, common to group VI TMDs. Therefore, we decided to develop parameters for $\mathrm{MoTe}_{2}$ rather than the more studied molybdenum disulfide $\left(\mathrm{MoS}_{2}\right)$, already explored by MD [54. Copper is the metal of choice for electrodes and metallic interconnects; hence, we studied the system $\mathrm{Cu}-\mathrm{MoTe}_{2}$. The following subsections describe the training data used in the force field parameterization and compare the optimized ReaxFF with the DFT training data.

\subsection{Initial parameters and charge equilibration}

We built an initial force field with parameters for Mo and Te extracted from Ref. [55] and Cu from Ref. [56]. The general parameters were re-optimized in order to improve the atomic features including equation of state and formation energy, as described in details in the following sections. Therefore, the parameters presented here are not transferable to the initial ReaxFF they belong to and the new force field we propose corresponds to a new branch of the ReaxFF tree, as defined in Ref. 40. Additionally, we introduce three anglar terms corresponding to Te-Mo-Te, $\mathrm{Te}-\mathrm{Cu}-\mathrm{Te}$ and $\mathrm{Cu}-\mathrm{Te}-\mathrm{Cu}$ in order to describe the subtle energy difference between $\mathrm{MoTe}_{2}$ phases and the potential energy landscape of $\mathrm{Cu}$ diffusion in $\mathrm{MoTe}_{2}$. We first optimized the QEq parameters, traditionally named $\gamma_{E E M}, \chi_{E E M}$ and $\eta_{E E M}$ representing the 
shielding distance for Coulomb interactions, electronegativity and hardness parameters, respectively. Partial atomic charges were optimized on each atom against Bader charges. We show in Table 1 the root mean square error of charges per atom computed between the optimized ReaxFF and DFT. ReaxFF describes accurately charges with an average error of $0.037 \pm 0.015 e$ per atom compared to Bader charges.

\subsection{Bulk phases and bond dissociation}

A key feature of ReaxFF lies in its ability to describe atoms in various chemical environments, corresponding to different coordinations. Therefore, we fit ReaxFF against various bulk phases of $\mathrm{Cu}$ and Mo including simple cubic (SC), face centered cubic (FCC) and body centered cubic (BCC). Figure 1 shows various equations of state (EOS) computed with DFT and ReaxFF, with minimum energy shifted to their corresponding enthalpies of formation (see Table 1 for details). For both $\mathrm{Mo}$ and $\mathrm{Cu}$, ReaxFF describes with accuracy the ground state structures, only the simple cubic phases present some discrepancy with respect to DFT. However, the simple cubic phases lie tens of $\mathrm{kcal} / \mathrm{mol}$ higher than the ground state and therefore do not represent important contribution to their chemistry; it is only important that they remain energetically prohibited.

$\mathrm{MoTe}_{2}$ has been observed in the stable $\mathrm{H}$ and $\mathrm{T}$ ' phases as well as a metastable $\mathrm{T}$ phase, corresponding to trigonal prismatic, distorted octahedral and octahedral geometries, respectively. The semiconducting $\mathrm{H}$ phase has been shown to be the ground state configuration for Mo-based TMDs while the metallic T' phase lies few $\mathrm{meV}$ above the $\mathrm{H}$ for $\mathrm{MoTe}_{2}$. A transition from $\mathrm{H}$ to $\mathrm{T}^{\prime}$ has been predicted from DFT calculations by mechanical strain in the range of 0.3 to $3 \%$ [17] or an applied electrostatic gate voltage [18]. Figure 2 shows the EOS corresponding to various phases of bulk $\mathrm{MoTe}_{2}$ computed with DFT and ReaxFF, and shifted to their binding energy. The binding energy is defined as the total energy of the $\mathrm{MoTe}_{2}$ phase minus the total energies of bulk Mo in its ground state $\mathrm{BCC}$ phase and the diatomic molecule $\mathrm{Te}_{2}$. The force field predicts the $\mathrm{H}$ phase to be the ground state however, the T' phase appears higher than the $\mathrm{T}$ phase. Additionally, we computed the potential energy surface corresponding to transition between $\mathrm{H} \rightarrow \mathrm{T}$ ' within the rectangular unit cell constrained to the initial $\mathrm{H}$ phase, as depicted in Figure 3 . We found that ReaxFF predicts the energy barrier between $\mathrm{H}$ and $\mathrm{T}$ ' phases $\mathrm{E}_{A}=32.3 \mathrm{kcal} / \mathrm{mol}$ in good agreement compared to $35.7 \mathrm{kcal} / \mathrm{mol}$ computed from DFT calculations. We note that ReaxFF is able to capture subtle energy differences between the various phases of $\mathrm{MoTe}_{2}$.

The bond order nature of ReaxFF enables dynamical connectivity during molecular dynamics simulations and the ability to describe dissociation (and formation) of chemical bonds. Therefore, the training set includes various bond dissociation curves, such as Te-Te and Mo-Te bonds in $\mathrm{Te}_{2}$ molecule and mono- 

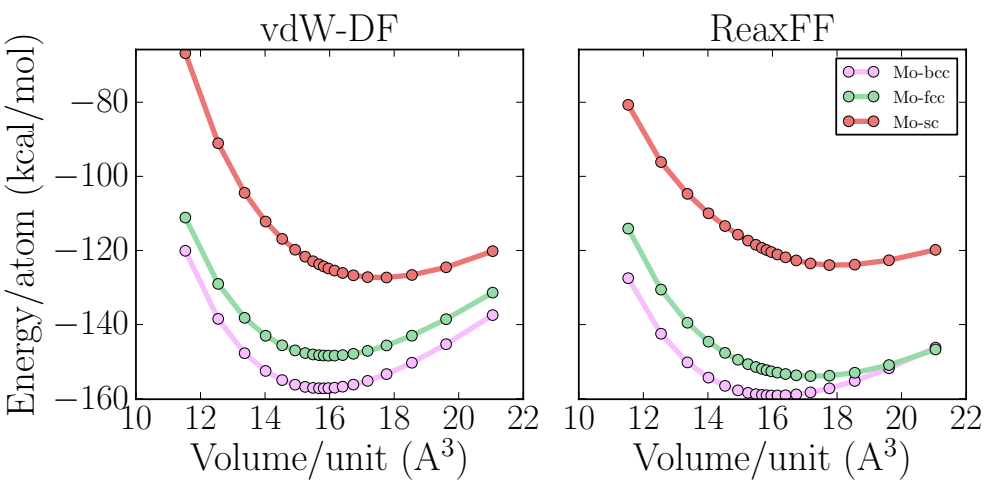

(a)
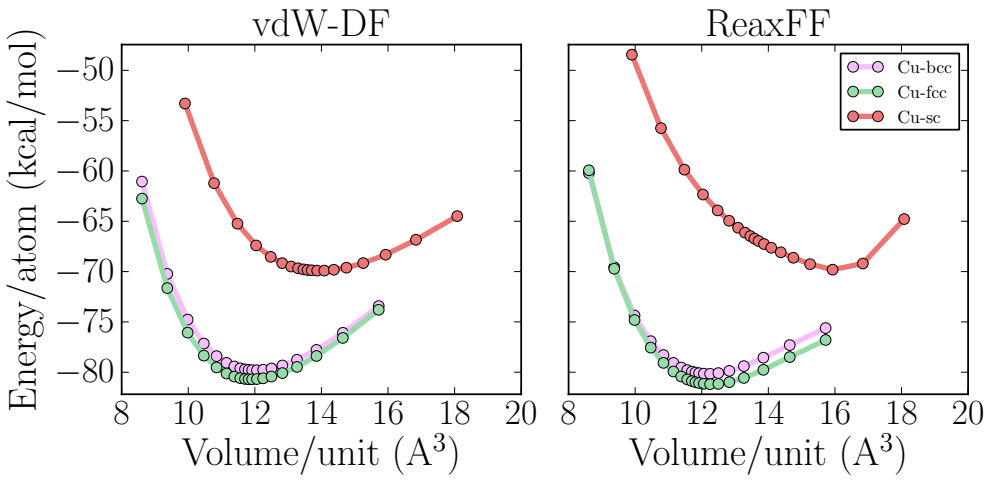

Figure 1: Equation of states for various bulk phases of $\mathrm{Mo}(\mathrm{a})$ and $\mathrm{Cu}(\mathrm{b})$ computed with vdW-DF (left) and ReaxFF (right) as a function of the volume of the unit cell. The minimum energy of the EOS have been shifted to the enthalpy of formation of the corresponding crystal.
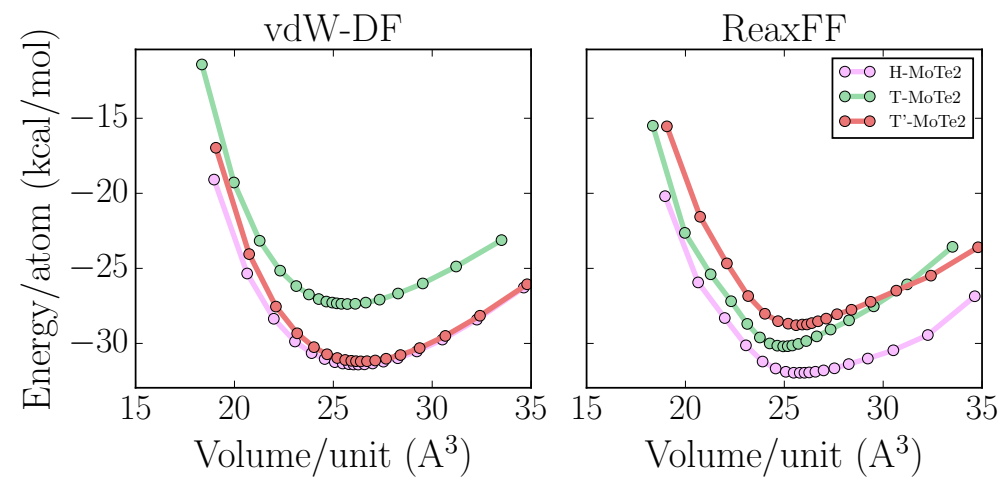

Figure 2: Equation of states for various bulk phases of $\mathrm{MoTe}_{2}$ computed with vdW-DF (left) and ReaxFF (right) as a function of the volume of the unit cell. The minimum energy of EOS have been shifted to the binding energy of the corresponding phase.

layer $\mathrm{MoTe}_{2}$, respectively. The corresponding energies versus bond distance plots are shown in Figure 4. We found a good agreement between ReaxFF and DFT even though the force field predicts slightly lower dissociation energies for both Te-Te and Mo-Te dissociations.

Finally, in order to validate the stability of the force field we performed molecular dynamics simulations 


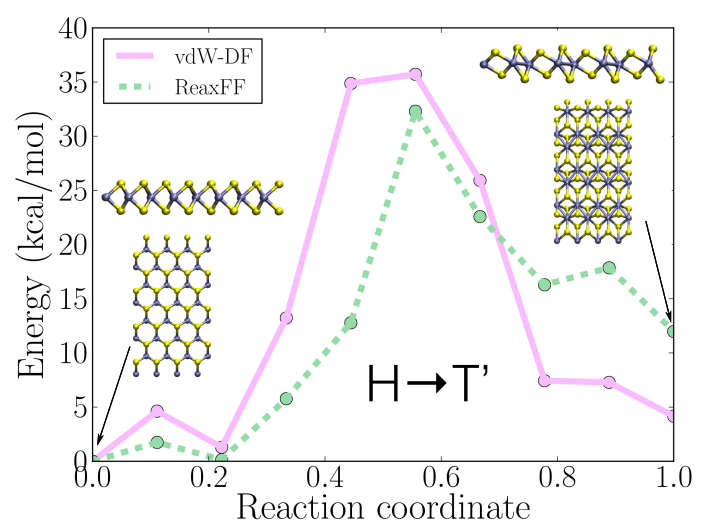

Figure 3: Potential energy surface corresponding to the transition of rectangular monolayer $\mathrm{MoTe}_{2}$ from the phase $\mathrm{H} \rightarrow \mathrm{T}^{\prime}$ computed with vdW-DF (solid) and ReaxFF (dashed) as a function of reaction coordinate.
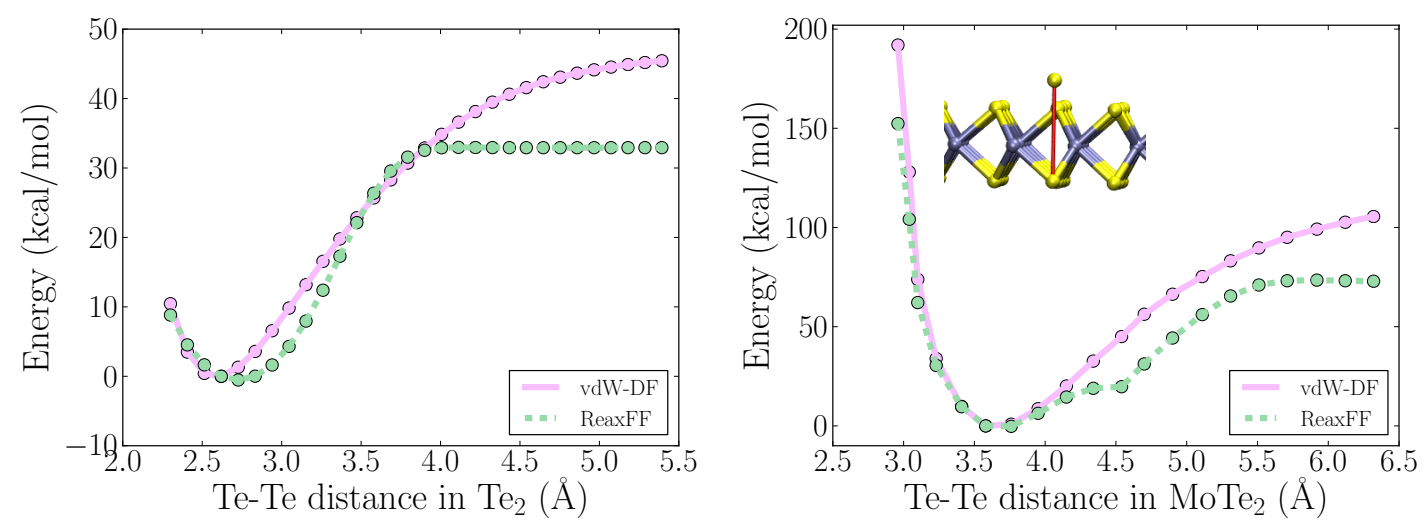

Figure 4: Dissociation curve of the $\mathrm{Te}_{2}$ molecule (left) and Te atom from $4 \times 4$ monolayer $\mathrm{MoTe}_{2}$ (left) computed with vdW-DF (solid) and ReaxFF (dashed) as a function of the corresponding bond distance.

of bulk and monolayer $\mathrm{MoTe}_{2}$ in the microcanonical ensemble with a timestep of $0.5 \mathrm{fs}$. We found little drift in the total energy $\left(\sim 1 \times 10^{-6} \mathrm{kcal} / \mathrm{mol} / \mathrm{ps} /\right.$ atom $)$ suggesting a good stability of the force field.

\subsection{Cu-intercalated $\mathrm{MoTe}_{2}$}

The intercalation of $\mathrm{Cu}$ atoms within the vdW gaps of ground state $\mathrm{H}-\mathrm{MoTe}_{2}$ can occur either at octahedral (or hollow, noted "h") or tetrahedral ("t") sites. Figure 5 shows the energetics of $h$ - (Fig. 5 5 ) and t- (Fig. 5) intercalated $\mathrm{Cu}_{x} \mathrm{MoTe}_{2}$ for $\mathrm{Cu}$ concentrations $x=0.125,0.25,0.375$ and 0.5 . We show energy as a function of volume upon uniaxial deformation along the direction perpendicular to the plane of the TMD in order to better test the interactions between $\mathrm{Cu}$ and the TMDs. The energy curves in Figure 5 are referenced to the perfect TMD and $\mathrm{Cu}$ as: $E_{f}^{C u}=E_{C u_{x} M o T e_{2}}-E_{M o T e_{2}}-x E_{C u}$ where $E_{C u_{x} M o T e_{2}}$, $E_{M o T e_{2}}$ and $E_{C u}$ are the energies of $\mathrm{Cu}_{x} \mathrm{MoTe}_{2}$, bulk $\mathrm{MoTe}_{2}$ and $\mathrm{Cu}$ in its ground state FCC phase, 
respectively.

The overall ReaxFF description of the structures and energetics is very accurate. The force field predicts the formation energy of the low concentration $\mathrm{Cu}$ h-intercalated $\mathrm{MoTe}_{2} \mathrm{E}_{f}^{0.125 h}=26.2 \mathrm{kcal} / \mathrm{mol}$, lower than the corresponding t-intercalated energy $\mathrm{E}_{f}^{0.125 t}=28.3 \mathrm{kcal} / \mathrm{mol}$, consistent with the predicted DFT energies of 27.2 and $28.2 \mathrm{kcal} / \mathrm{mol}$, respectively. However, our force field does not describe with accuracy the trends of formation energy corresponding to $h$-intercalated $\mathrm{Cu}-\mathrm{MoTe}_{2}$ systems. The intercalation of $\mathrm{Cu}$ in h-sites $\mathrm{MoTe}_{2}$ requires a narrow range of energies between 27.2 to $29.1 \mathrm{kcal} / \mathrm{mol}$, which is challenging to be resolved by the force field.
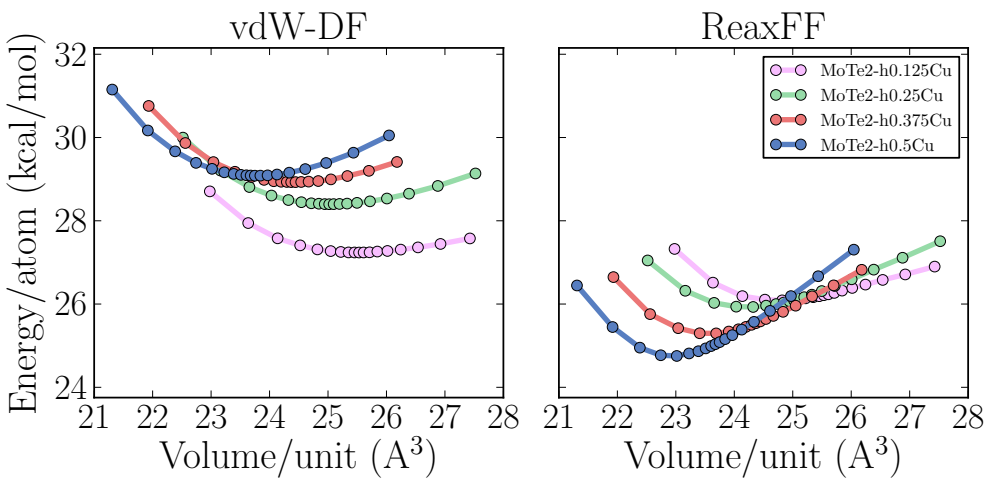

(a)
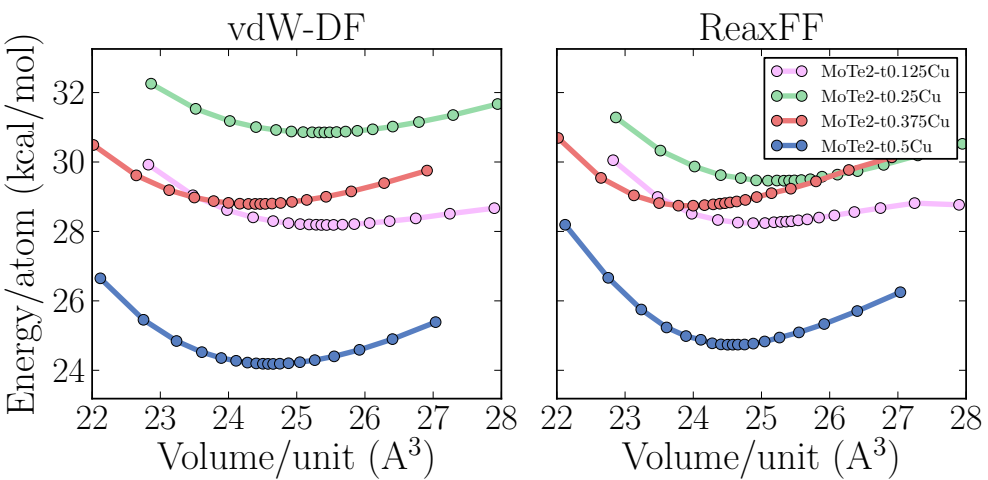

(b)

Figure 5: Energies corresponding to uniaxial deformation along the axis perpendicular to the $\mathrm{MoTe}_{2}$ layer for various concentration of $\mathrm{Cu}$ intercalated at $h$ - and $t$-sites computed with vdW-DF (left) and ReaxFF (right) as a function of the volume of the unit cell.

Figure 6 shows the potential energy surface corresponding to migration of $\mathrm{Cu}$ between neighboring octahedral sites as well as $2^{\text {nd }}$ neighbor octahedral sites in bulk $\mathrm{MoTe}_{2}$. From DFT calculations, we found the energy barriers for $\mathrm{Cu}$ diffusion from octahedral to tetrahedral and between two tetrahedral sites $\mathrm{E}_{A}^{h \rightarrow t}=9.2 \mathrm{kcal} / \mathrm{mol}$ and $\mathrm{E}_{A}^{t \rightarrow t}=13.1 \mathrm{kcal} / \mathrm{mol}$, respectively. The force field slightly over estimates the energy barriers $\mathrm{E}_{A}^{h \rightarrow t}=11.5 \mathrm{kcal} / \mathrm{mol}$ and $\mathrm{E}_{A}^{t \rightarrow t}=20.2 \mathrm{kcal} / \mathrm{mol}$.

In order to capture the proper dynamics of $\mathrm{Cu}$ diffusion and clusterization inside the vdW gap of 

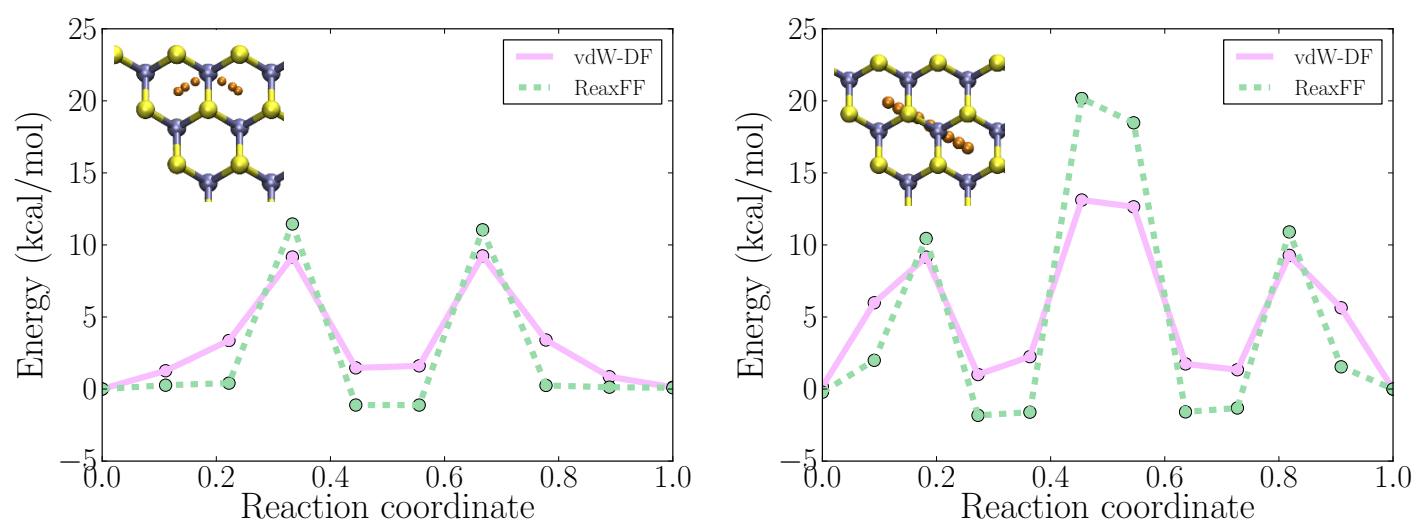

Figure 6: Potential energy surface corresponding to the migration of a copper atom within the vdW gap of $4 \times 4$ bulk $\mathrm{MoTe}_{2}$ computed with vdW-DF (solid) and ReaxFF (dashed) as a function of reaction coordinate. $\mathrm{Cu}$ diffusion between neighboring octahedral sites through a tetrahedral site (left) and $\mathrm{Cu}$ diffusion between $2^{\text {nd }}$ neighbor octahedral sites via two tetrahedral sites.

$\mathrm{MoTe}_{2}$, we performed ab initio MD simulations of $\mathrm{Cu}_{3}$ cluster intercalated $\mathrm{MoTe}_{2}$. As shown in the inset of Figure 7, we start the $\mathrm{MD}$ simulation with a $\mathrm{Cu}_{3}$ cluster located around a tetragonal site with each atom in the cluster seating in the corner of an octahedral site. MD simulation at $500 \mathrm{~K}$ shows the dissociation of the $\mathrm{Cu}_{3}$ cluster into three isolated $\mathrm{Cu}$ atoms moving away from each other toward an opposite tetrahedral site. Nine snapshots along the dissociation have been relaxed with DFT constraining the $x y$ (in-plane) dimensions of the $\mathrm{Cu}$ atoms and we extracted the potential energy surface presented in Figure 7. DFT calculations predict that $\mathrm{Cu}_{3}$ needs to overcome an energy barrier $E_{A}=28.1 \mathrm{kcal} / \mathrm{mol}$ for dissociation. On the other hand, ReaxFF overestimates the barrier by a factor of 1.75 leading to an activation energy $E_{A}=49.4 \mathrm{kcal} / \mathrm{mol}$. Moreover, we compare on Figure 7 (right panel) the charges on each atom along the dissociation of the $\mathrm{Cu}_{3}$ cluster computed with Bader analysis and ReaxFF's QEq. Charges on each atom are roughly constant and we found a very good agreement between charges computed with the force field and DFT.

To summarize, we report on Table 1 the root mean square error per atom corresponding to charges, forces and pressure computed between DFT and ReaxFF as well as the various formation energies. We acknowledge that the force field presents some uncertainties, and the main deviations, with respect to DFT calculations, are related to the energy barriers for $\mathrm{Cu}$ diffusion and cluster dissociation. The overestimation of diffusion barriers suggest a slower mobility of $\mathrm{Cu}$ atoms described by ReaxFF than predicted from DFT. We provide the optimized ReaxFF parameters in Supplementary Materials. 

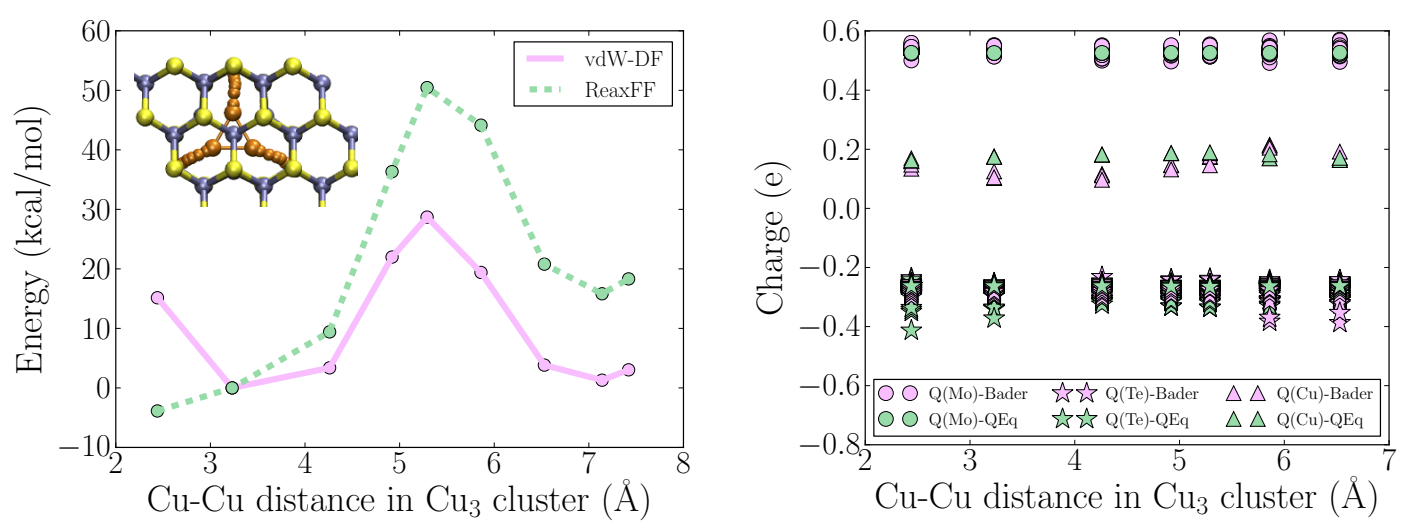

Figure 7: Potential energy surface corresponding to the dissociation of a copper cluster $\mathrm{Cu}_{3}$ intercalated inside the vdW gap of $4 \times 4$ bulk $\mathrm{MoTe}_{2}$ computed with vdW-DF and ReaxFF (left) and the corresponding Bader and QEq partial charges (right) as a function of the average $\mathrm{Cu}-\mathrm{Cu}$ distance in the $\mathrm{Cu}_{3}$ cluster.

\subsection{Mechanical properties}

In addition to the energetics analysis, we evaluated the capacity of ReaxFF to describe the mechanical properties of the structures included in the training set. Equilibrium volumes, bulk moduli and elastic constants are reported on Table 1, computed with both DFT and ReaxFF. We found between 15 and $52 \%$ error in the description of bulk moduli for bulk $\mathrm{Cu}$ and Mo. However, since the main purpose of the presented force field lies in the description of $\mathrm{Cu}$-intercalated $\mathrm{MoTe}_{2}$, we consider this large error acceptable. By contrast, ReaxFF describes with great accuracy the moduli corresponding to various $\mathrm{MoTe}_{2}$ phases. Moreover, ReaxFF describes the gradual stiffening of intercalated $\mathrm{MoTe}_{2}$ with increase number of $\mathrm{Cu}$ intercalant, consistent with DFT calculations.

\section{Force field validation: $\mathrm{Cu} / \mathrm{MoTe}_{2}$ interface}

We used the force field to compute the interaction between a single layer of $\mathrm{MoTe}_{2}$ and a (111) $\mathrm{Cu}$ surface. We follow the strategy developed in Ref. 57 to define supercells and minimize strain, chosen to be applied to the metal slab because of the corresponding small effect on the electronic structure of the interface [25]. We develop the in-plane supercell lattice vectors $\vec{T}_{1}=n_{1} \vec{a}_{1}+n_{2} \vec{a}_{2}$ and $\vec{T}_{1}^{\prime}=m_{1} \vec{b}_{1}+m_{2} \vec{b}_{2}$ over the primitive hexagonal cells of $\mathrm{MoTe}_{2}\left\{\vec{a}_{1}, \vec{a}_{2}\right\}$ and the $\mathrm{Cu}$ slab $\left\{\vec{b}_{1}, \vec{b}_{2}\right\}$, respectively. From the optimized lattice parameters of monolayer $\mathrm{MoTe}_{2}$ and $\mathrm{Cu}$ slab computed with both DFT and ReaxFF, we minimize the lattice mismatch $\delta=\left(\left|\vec{T}_{1}\right|-\left|\vec{T}_{1}^{\prime}\right|\right) /\left|\vec{T}_{1}\right|$ and select two interfaces with equivalent strain, as reported on Table 2

Figure 8 shows the adhesion energy (the energy of the interface minus the energy of the $\mathrm{Cu}$ slab 
Table 1: Root mean square error per atom (Err.) between the DFT (vdW-DF) and ReaxFF charges $(\mathrm{Q})$, forces $(\mathrm{f})$ and pressures $(\mathrm{P})$ as well as formation energies $\left(\mathrm{E}_{f}\right)$, equilibrium volumes $\left(\mathrm{V}_{0}\right)$ and elastic properties (bulk moduli $\mathrm{B}_{0}$ and elastic constants $\mathrm{C}_{11}$ ) for various compounds included in the training set.

\begin{tabular}{|c|c|c|c|c|c|c|}
\hline \multirow[t]{2}{*}{ Crystal/molecule } & \multirow[t]{2}{*}{$\begin{array}{l}\text { Err. } Q^{*} \\
\text { (e) }\end{array}$} & \multirow[t]{2}{*}{$\begin{array}{c}\text { Err. } \mathrm{f}^{*} \\
(\mathrm{kcal} / \mathrm{mol} / \AA)\end{array}$} & \multirow[t]{2}{*}{$\begin{array}{l}\text { Err. P* } \\
\text { (Atm.) }\end{array}$} & $\begin{array}{c}\mathrm{E}_{f}^{\star} \\
(\mathrm{kcal} / \mathrm{mol})\end{array}$ & $\begin{array}{c}\mathrm{V}_{0}^{\dagger} \\
\left(\AA^{3}\right)\end{array}$ & $\begin{array}{c}\mathrm{B}_{0}^{\dagger} \text { or } \mathrm{C}_{11}^{\ddagger} \\
(\mathrm{GPa})\end{array}$ \\
\hline & & & & \multicolumn{3}{|c|}{ vdW-DF / ReaxFF } \\
\hline $\mathrm{Cu} \mathrm{bcc}$ & - & - & 6.35 & -79.8/-80.1 & $1212.0 / 12.3$ & 135.2/116.5 \\
\hline $\mathrm{Cu}$ fcc & - & - & 11.42 & $-80.7 /-81.1$ & $11.9 / 12.4$ & $139.5 / 117.9$ \\
\hline $\mathrm{Cu} \mathrm{sc}$ & - & - & 18.96 & $-69.9 /-67.6$ & $13.9 / 15.4$ & $101.7 / 104.2$ \\
\hline Mo bcc & - & - & 18.05 & $-157.2 /-159.1$ & $15.8 / 16.1$ & $259.8 / 189.6$ \\
\hline Mo fcc & - & - & 21.12 & $-148.4 /-152.6$ & $16.0 / 17.2$ & $237.9 / 156.5$ \\
\hline Mo sc & - & - & 39.39 & $-127.3 /-124.0$ & $17.6 / 18.2$ & $197.2 / 145.7$ \\
\hline $\mathrm{MoCu}(\mathrm{CsCl})$ & 0.06 & 0.00 & 10.57 & $\mathrm{X}$ & $26.5 / 26.3$ & $48.2 / 47.2$ \\
\hline $\mathrm{MoTe}_{2} \mathrm{H}+0.125 \mathrm{Cu}_{h}$ & 0.03 & 0.88 & 2.50 & $27.2 / 26.2$ & $25.8 / 25.2$ & $2.4 / 2.6^{d}$ \\
\hline $\mathrm{MoTe}_{2} \mathrm{H}+0.25 \mathrm{Cu}_{h}$ & 0.03 & 1.20 & 2.64 & $28.4 / 26.1$ & $25.3 / 24.5$ & $2.6 / 2.6^{d}$ \\
\hline $\mathrm{MoTe}_{2} \mathrm{H}+0.375 \mathrm{Cu}_{h}$ & 0.03 & 1.12 & 3.63 & $28.9 / 25.6$ & $24.6 / 23.8$ & $3.4 / 4.0^{d}$ \\
\hline $\mathrm{MoTe}_{2} \mathrm{H}+0.5 \mathrm{Cu}_{h}$ & 0.04 & 1.06 & 4.60 & $29.1 / 25.1$ & $24.0 / 23.2$ & $3.7 / 4.6^{d}$ \\
\hline $\mathrm{MoTe}_{2} \mathrm{H}+0.125 \mathrm{Cu}_{t}$ & 0.03 & 1.42 & 2.42 & $28.2 / 28.3$ & $25.9 / 25.7$ & $2.4 / 2.4^{d}$ \\
\hline $\mathrm{MoTe}_{2} \mathrm{H}+0.25 \mathrm{Cu}_{t}$ & 0.04 & 1.72 & 1.44 & $30.9 / 29.5$ & $25.6 / 25.6$ & $2.4 / 3.1^{d}$ \\
\hline $\mathrm{MoTe}_{2} \mathrm{H}+0.375 \mathrm{Cu}_{t}$ & 0.04 & 2.18 & 1.50 & $28.8 / 28.8$ & $24.7 / 24.4$ & $3.1 / 3.7^{d}$ \\
\hline $\mathrm{MoTe}_{2} \mathrm{H}+0.5 \mathrm{Cu}_{t}$ & 0.04 & 2.46 & 2.55 & $24.2 / 24.7$ & $24.9 / 24.9$ & $4.2 / 5.8^{d}$ \\
\hline $\mathrm{MoTe}_{2} \mathrm{H}$ & 0.04 & 1.51 & 5.26 & $-31.4 /-31.9$ & $26.2 / 26.4$ & $45.3 / 43.6$ \\
\hline $\mathrm{MoTe}_{2} \mathrm{~T}$ & 0.06 & 1.21 & 6.53 & $-27.4 /-30.0$ & $25.8 / 25.5$ & $47.3 / 58.0$ \\
\hline $\mathrm{MoTe}_{2} \mathrm{~T}^{\prime}$ & 0.06 & 4.46 & 6.97 & $-31.2 /-28.6$ & $26.5 / 26.3$ & $48.2 / 47.2$ \\
\hline $\mathrm{MoTe}_{2} 3 \mathrm{Cu}$ diss & 0.03 & 1.02 & 1.47 & $\mathrm{X}$ & $\mathrm{X}$ & $\mathrm{X}$ \\
\hline $\mathrm{MoTe}_{2}$ diss & 0.05 & 1.18 & 0.70 & - & - & - \\
\hline $\mathrm{Te}_{2}$ diss & 0.00 & 2.99 & 0.00 & - & - & - \\
\hline $\mathrm{NEB} \mathrm{H} \rightarrow \mathrm{T}^{\prime}$ & 0.05 & 2.72 & 2.03 & $\mathrm{X}$ & $\mathrm{X}$ & $\mathrm{X}$ \\
\hline NEB path1 & 0.02 & 0.49 & 1.35 & - & - & - \\
\hline NEB path2 & 0.02 & 0.56 & 1.29 & - & - & - \\
\hline
\end{tabular}

${ }^{*} \mathrm{RMSE}_{i}=\sqrt{\left(i^{\mathrm{vdW}-\mathrm{DF}}-i^{\mathrm{ReaxFF}}\right)^{2} / N_{i}^{2}}$

${ }^{\star}$ Example for $\mathrm{MX}_{2}: E_{f}=E_{M X_{2}}-\left(E_{M}^{0}+2 E_{X}^{0}\right)$

${ }^{\dagger}$ From Murnaghan equation

$\ddagger$ From parabola $E=E_{0}+C_{11} V^{2} / 2$

Table 2: Parameters corresponding to the supercell construction of a $\mathrm{Cu} / \mathrm{MoTe}_{2}$ interface with minimal lattice mismatch $(\delta)$. The two sets $\left(n_{1}, n_{2}\right)$ and $\left(m_{1}, m_{2}\right)$ correspond to the coefficients of the in-plane supercells for $\mathrm{MoTe}_{2}$ and $\mathrm{Cu}$, respectively. We note that the two supercells have been rotated by the angle $\alpha$.

\begin{tabular}{c|cccc} 
Method & $n_{1}, n_{2}$ & $m_{1}, m_{2}$ & $\alpha\left(^{\circ}\right)$ & $\delta(\%)$ \\
\hline \hline ReaxFF & 5,4 & 7,5 & 1.8 & 1.5 \\
vdw-DF & 2,1 & 3,1 & 5.2 & 1.3 \\
\hline
\end{tabular}

minus the energy of the $\mathrm{MoTe}_{2}$ layer) computed with DFT and ReaxFF. We find very good agreement between the force field and DFT calculations even though such data was not included in the force field 


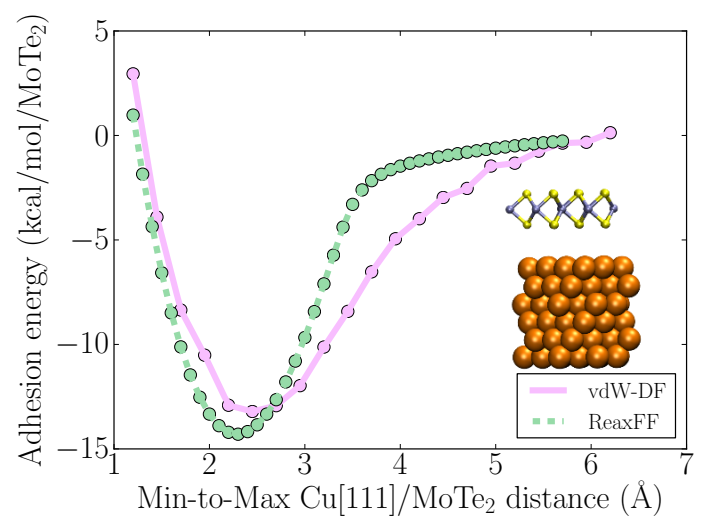

Figure 8: Adhesion energy as a function of the minimum separation between a slab of $\mathrm{Cu}(111)$ and $\mathrm{MoTe}_{2}$ computed with vdW-DF (solid) and ReaxFF (dashed).

parameterization. This indicates the type of regimes the force field is expected to be extrapolated to. However, as with any force field, care should be exercised when using it away for the conditions for which it was trained.

\section{Diffusion of Cu-intercalated $\mathrm{MoTe}_{2}$}

The relatively small computational intensity of ReaxFF enables the simulations of large scale systems; providing a unique tool for exploring ionic transport, fracture and the effect of defects on mechanical properties and growth. In this section we focus on the first of these examples.

We know from the first principle calculations presented above that the activation energy for thermal hop of $\mathrm{Cu}$ intercalated $\mathrm{MoTe}_{2}$ varies between 9-13 kcal/mol (0.39-0.56 eV) range, depending on the intercalation site. These values are intermediate between the extreme cases of a good ion conductor like highly metal doped chalcogenide glasses with $\mathrm{E}_{A} \sim 0.2 \mathrm{eV}[58$ ] and amorphous silica with in average $\mathrm{E}_{A} \sim 0.8 \mathrm{eV}$ [59]. In order to confirm this last statement, we performed simulations of Cu-intercalated bulk $\mathrm{MoTe}_{2}$ at various temperature ranging from 340 to $380 \mathrm{~K}$ and evaluated the diffusion coefficient $\mathrm{D} \approx$ $\left[10^{-7}-10^{-8}\right] \mathrm{cm}^{2} / \mathrm{s}$ (see Supplementary Materials). Therefore, within the timescales of MD and at room temperature we find negligible thermal diffusion; thus we study the drift of the ions under an external bias, relevant to estimate the electromobility of $\mathrm{Cu}$-intercalated ions in $\mathrm{MoTe}_{2}$-based device operation.

We now explore the effect of $\mathrm{Cu}$ concentration on ion mobility in $\mathrm{Cu}$-intercalated bulk $\mathrm{MoTe}_{2}$ under the action of an external electric field. We performed MD simulations of $\mathrm{Cu}$-intercalated bulk $\mathrm{H}-\mathrm{MoTe}_{2}$ in the canonical ensemble at $300 \mathrm{~K}$ for up to $2 \mathrm{~ns}$ and applied various external electric fields (i.e. a constant force $\mathrm{F}_{i}=\mathrm{Eq}_{i}$ to each atom $i$ ) ranging from 0.0 to $0.6 \mathrm{~V} / \AA$. The direction of the electric field 
is chosen to be aligned with the in-plane lattice parameter of the hexagonal cell of $\mathrm{MoTe}_{2}$, as shown in the inset of Figure 9. We varied the concentration of $\mathrm{Cu}$ ions from 1 to $4 \%$ with respect to $\mathrm{MoTe}_{2}$ and study their mobility. In order to estimate uncertainties in the mobility of the $\mathrm{Cu}$ ions, we performed simulations over an ensemble of 4 independent samples differing by the initial position and velocity of copper atoms.

Since thermal diffusion leads to no net motion of the atoms, the average position of the $\mathrm{Cu}$ ions as a function of time $<X(t)>$, can be described as:

$$
<X(t)>=X_{0}+v_{d} t
$$

The mobility $u$ can then be obtained from the applied electric field $E$ and drift velocity $u=v_{d} / E[60$. Figure 9 shows the computed drift velocity of $\mathrm{Cu}$-intercalated $\mathrm{MoTe}_{2}$ as a function of the electric field (left) and the $\mathrm{Cu}$ fraction (right). We found that a minimum electric field of approximately $0.4 \mathrm{~V} / \AA$ is required to displace the $\mathrm{Cu}$ ions in the $\mathrm{vdW}$ gap of $\mathrm{MoTe}_{2}$ within MD timescales. More importantly, we find that the ionic mobility increases with concentration. Finally, since ReaxFF predicts slightly higher diffusion barriers, with respect to DFT calculations, we expect the electric field threshold for drift diffusion to be lower than the $0.4 \mathrm{~V} / \AA$ p predicted here.
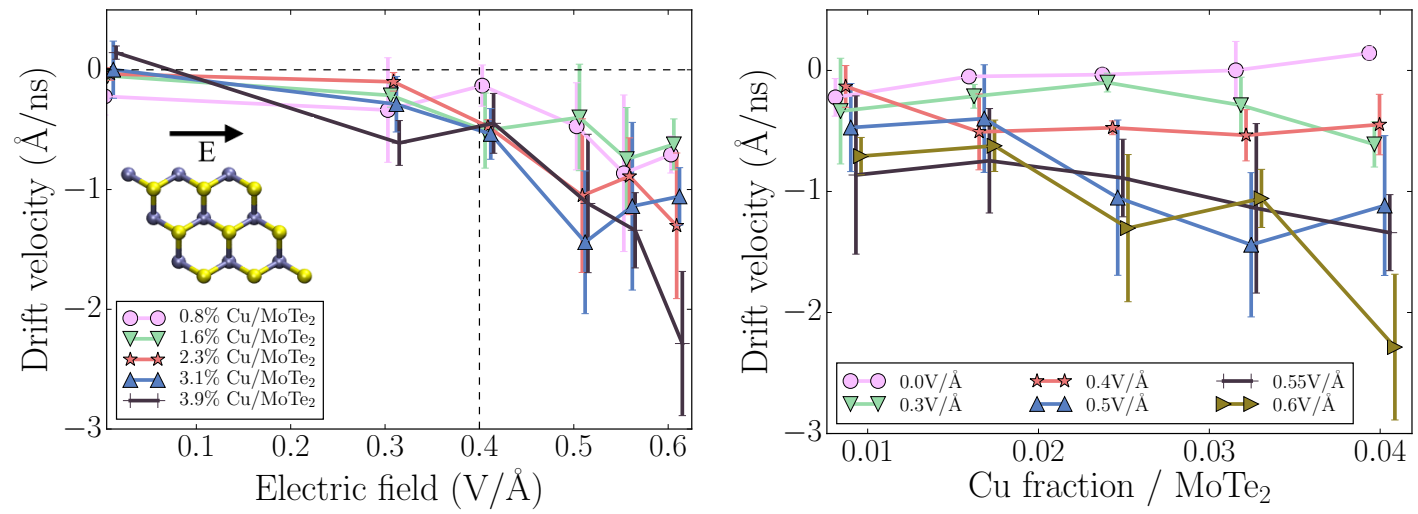

Figure 9: Drift velocity of $\mathrm{Cu}$ ions as a function of the electric field for various $\mathrm{Cu}$ fraction (left) and as a function of the $\mathrm{Cu}$ fraction for various electric field (right). The error bars have been evaluated from the standard deviation of drift velocity over 4 independent samples and the data points between curves have been slightly shifted in order to visualize the error bars. 


\section{Conclusions}

We presented a parameterization of ReaxFF to describe the interactions between $\mathrm{MoTe}_{2}$ and copper and showed that such force field can be used to study large scale atomistic simulations of interest for emerging electronics. The optimization of the force field against first principle calculations shows that ReaxFF reproduces with accuracy the energetics, charges and mechanical properties of various systems composed of $\mathrm{MoTe}_{2}$ and $\mathrm{Cu}$ and slightly overestimates the energy barrier for $\mathrm{Cu}$ diffusion in the vdW gap of the TMD. Additionally, we demonstrated the good transferability of the force field to describe $\mathrm{Cu} / \mathrm{MoTe}_{2}$ interfaces. As for any force field, care must be taken when used outside of the conditions used during parameterization. For example, the force field has not been optimized to describe defect formation energies and extensive validations must be performed in order to study growth, as we envisage in the future.

\section{Acknowledgement}

We acknowledge Benjamin Helfrecht for DFT calculations of the binding energy between $\mathrm{Cu}$ and $\mathrm{MoTe}_{2}$. This work was partially supported by the FAME and LEAST Centers, two of six centers of STARnet, a Semiconductor Research Corporation program sponsored by MARCO and DARPA. We thank nanoHUB.org and Purdue for the computational resources. 


\section{References}

[1] Branimir Radisavljevic, Aleksandra Radenovic, Jacopo Brivio, V Giacometti, and A Kis. Single-layer MoS2 transistors. Nature Nanotechnology, 6(3):147-150, 2011.

[2] Deep Jariwala, Vinod K Sangwan, Lincoln J Lauhon, Tobin J Marks, and Mark C Hersam. Emerging device applications for semiconducting two-dimensional transition metal dichalcogenides. ACS Nano, 8(2):1102-1120, 2014.

[3] Saptarshi Das, Abhijith Prakash, Ramon Salazar, and Joerg Appenzeller. Toward low-power electronics: tunneling phenomena in transition metal dichalcogenides. ACS Nano, 8(2):1681-1689, 2014.

[4] Manish Chhowalla, Hyeon Suk Shin, Goki Eda, Lain-Jong Li, Kian Ping Loh, and Hua Zhang. The chemistry of two-dimensional layered transition metal dichalcogenide nanosheets. Nature Chemistry, $5(4): 263-275,2013$.

[5] E Morosan, HW Zandbergen, BS Dennis, JWG Bos, Y Onose, T Klimczuk, AP Ramirez, NP Ong, and RJ Cava. Superconductivity in CuxTiSe2. Nature Physics, 2(8):544-550, 2006.

[6] Haotian Wang, Hongtao Yuan, Seung Sae Hong, Yanbin Li, and Yi Cui. Physical and chemical tuning of two-dimensional transition metal dichalcogenides. Chemical Society Reviews, 44(9):2664$2680,2015$.

[7] Hannu-Pekka Komsa, Jani Kotakoski, Simon Kurasch, Ossi Lehtinen, Ute Kaiser, and Arkady V Krasheninnikov. Two-dimensional transition metal dichalcogenides under electron irradiation: defect production and doping. Physical Review Letters, 109(3):035503, 2012.

[8] Jung Ho Yu, Hye Ryoung Lee, Seung Sae Hong, Desheng Kong, Hyun-Wook Lee, Haotian Wang, Feng Xiong, Shuang Wang, and Yi Cui. Vertical heterostructure of two-dimensional MoS2 and WSe2 with vertically aligned layers. Nano Letters, 15(2):1031-1035, 2015.

[9] Adrien Allain, Jiahao Kang, Kaustav Banerjee, and Andras Kis. Electrical contacts to twodimensional semiconductors. Nature Materials, 14(12):1195-1205, 2015.

[10] Hui Fang, Steven Chuang, Ting Chia Chang, Kuniharu Takei, Toshitake Takahashi, and Ali Javey. High-performance single layered WSe2 p-fets with chemically doped contacts. Nano Letters, 12(7):3788-3792, 2012. 
[11] Wei Liu, Jiahao Kang, Deblina Sarkar, Yasin Khatami, Debdeep Jena, and Kaustav Banerjee. Role of metal contacts in designing high-performance monolayer n-type WSe2 field effect transistors. Nano Letters, 13(5):1983-1990, 2013.

[12] Junhao Lin, Ovidiu Cretu, Wu Zhou, Kazu Suenaga, Dhiraj Prasai, Kirill I Bolotin, Nguyen Thanh Cuong, Minoru Otani, Susumu Okada, Andrew R Lupini, et al. Flexible metallic nanowires with self-adaptive contacts to semiconducting transition-metal dichalcogenide monolayers. Nature Nanotechnology, 9(6):436-442, 2014.

[13] Ruchit Mehta, Sunny Chugh, and Zhihong Chen. Enhanced electrical and thermal conduction in graphene-encapsulated copper nanowires. Nano Letters, 15(3):2024-2030, 2015.

[14] Simone Bertolazzi, Daria Krasnozhon, and Andras Kis. Nonvolatile memory cells based on MoS2/graphene heterostructures. ACS Nano, 7(4):3246-3252, 2013.

[15] Alexander A Bessonov, Marina N Kirikova, Dmitrii I Petukhov, Mark Allen, Tapani Ryhänen, and Marc JA Bailey. Layered memristive and memcapacitive switches for printable electronics. Nature Materials, 14(2):199-204, 2015.

[16] Chaoliang Tan, Zhengdong Liu, Wei Huang, and Hua Zhang. Non-volatile resistive memory devices based on solution-processed ultrathin two-dimensional nanomaterials. Chemical Society Reviews, 44(9):2615-2628, 2015.

[17] Karel-Alexander N Duerloo, Yao Li, and Evan J Reed. Structural phase transitions in twodimensional Mo-and W-dichalcogenide monolayers. Nature Communications, 5, 2014.

[18] Yao Li, Karel-Alexander N Duerloo, Kerry Wauson, and Evan J Reed. Structural semiconductorto-semimetal phase transition in two-dimensional materials induced by electrostatic gating. Nature Communications, 7, 2016.

[19] Cheng Gong, Chunming Huang, Justin Miller, Lanxia Cheng, Yufeng Hao, David Cobden, Jiyoung Kim, Rodney S Ruoff, Robert M Wallace, Kyeongjae Cho, et al. Metal contacts on physical vapor deposited monolayer MoS2. ACS Nano, 7(12):11350-11357, 2013.

[20] Rajesh Kappera, Damien Voiry, Sibel Ebru Yalcin, Brittany Branch, Gautam Gupta, Aditya D Mohite, and Manish Chhowalla. Phase-engineered low-resistance contacts for ultrathin MoS2 transistors. Nature Materials, 13(12):1128-1134, 2014. 
[21] Stefan Grimme, Jens Antony, Stephan Ehrlich, and Helge Krieg. A consistent and accurate ab initio parametrization of density functional dispersion correction (DFT-D) for the 94 elements H-Pu. The Journal of Chemical Physics, 132(15):154104, 2010.

[22] Kyuho Lee, Éamonn D Murray, Lingzhu Kong, Bengt I Lundqvist, and David C Langreth. Higheraccuracy van der Waals density functional. Physical Review B, 82(8):081101, 2010.

[23] Jiahao Kang, Wei Liu, and Kaustav Banerjee. High-performance MoS2 transistors with lowresistance molybdenum contacts. Applied Physics Letters, 104(9):093106, 2014.

[24] Jiahao Kang, Wei Liu, Deblina Sarkar, Debdeep Jena, and Kaustav Banerjee. Computational study of metal contacts to monolayer transition-metal dichalcogenide semiconductors. Physical Review X, 4(3):031005, 2014.

[25] Mojtaba Farmanbar and Geert Brocks. First-principles study of van der Waals interactions and lattice mismatch at MoS2/metal interfaces. Physical Review B, 93(8):085304, 2016.

[26] Arend M van der Zande, Pinshane Y Huang, Daniel A Chenet, Timothy C Berkelbach, YuMeng You, Gwan-Hyoung Lee, Tony F Heinz, David R Reichman, David A Muller, and James C Hone. Grains and grain boundaries in highly crystalline monolayer molybdenum disulphide. Nature Materials, 12(6):554-561, 2013.

[27] Seok Joon Yun, Sang Hoon Chae, Hyun Kim, Jin Cheol Park, Ji-Hoon Park, Gang Hee Han, Joo Song Lee, Soo Min Kim, Hye Min Oh, Jinbong Seok, et al. Synthesis of centimeter-scale monolayer tungsten disulfide film on gold foils. ACS Nano, 9(5):5510-5519, 2015.

[28] Hye Yun Jeong, Si Young Lee, Thuc Hue Ly, Gang Hee Han, Hyun Kim, Honggi Nam, Zhao Jiong, Bong Gyu Shin, Seok Joon Yun, Jaesu Kim, et al. Visualizing point defects in transition-metal dichalcogenides using optical microscopy. ACS Nano, 10(1):770-777, 2015.

[29] Damien Voiry, Maryam Salehi, Rafael Silva, Takeshi Fujita, Mingwei Chen, Tewodros Asefa, Vivek B Shenoy, Goki Eda, and Manish Chhowalla. Conducting MoS2 nanosheets as catalysts for hydrogen evolution reaction. Nano Letters, 13(12):6222-6227, 2013.

[30] Li-ping Feng, Jie Su, Da-peng Li, and Zheng-tang Liu. Tuning the electronic properties of Ti-MoS2 contacts through introducing vacancies in monolayer MoS2. Physical Chemistry Chemical Physics, 17(10):6700-6704, 2015. 
[31] Zhong Lin, Bruno R Carvalho, Ethan Kahn, Ruitao Lv, Rahul Rao, Humberto Terrones, Marcos A Pimenta, and Mauricio Terrones. Defect engineering of two-dimensional transition metal dichalcogenides. 2D Materials, 3(2):022002, 2016.

[32] Adri CT van Duin, Alejandro Strachan, Shannon Stewman, Qingsong Zhang, Xin Xu, and William A Goddard. ReaxFFsio reactive force field for silicon and silicon oxide systems. The Journal of Physical Chemistry A, 107(19):3803-3811, 2003.

[33] Kevin D Nielson, Adri CT van Duin, Jonas Oxgaard, Wei-Qiao Deng, and William A Goddard. Development of the ReaxFF reactive force field for describing transition metal catalyzed reactions, with application to the initial stages of the catalytic formation of carbon nanotubes. The Journal of Physical Chemistry A, 109(3):493-499, 2005.

[34] Mitchell A Wood, Adri CT van Duin, and Alejandro Strachan. Coupled thermal and electromagnetic induced decomposition in the molecular explosive $\alpha \mathrm{HMX}$; a reactive molecular dynamics study. The Journal of Physical Chemistry A, 118(5):885-895, 2014.

[35] Mitchell A Wood and Alejandro Strachan. Non-equilibrium reaction kinetics in molecular solids. The Journal of Physical Chemistry C, 120(1):542-552, 2015.

[36] Mitchell A Wood, Mathew J Cherukara, Edward M Kober, and Alejandro Strachan. Ultrafast chemistry under non-equilibrium conditions and the shock to deflagration transition at the nanoscale. The Journal of Physical Chemistry C, 119(38):22008-22015, 2015.

[37] Adri CT van Duin, Siddharth Dasgupta, Francois Lorant, and William A Goddard. ReaxFF: a reactive force field for hydrocarbons. The Journal of Physical Chemistry A, 105(41):9396-9409, 2001.

[38] Wilfried J Mortier, Swapan K Ghosh, and S Shankar. Electronegativity-equalization method for the calculation of atomic charges in molecules. Journal of the American Chemical Society, 108(15):4315$4320,1986$.

[39] Anthony K Rappe and William A Goddard III. Charge equilibration for molecular dynamics simulations. The Journal of Physical Chemistry, 95(8):3358-3363, 1991.

[40] Thomas P Senftle, Sungwook Hong, Md Mahbubul Islam, Sudhir B Kylasa, Yuanxia Zheng, Yun Kyung Shin, Chad Junkermeier, Roman Engel-Herbert, Michael J Janik, Hasan Metin Aktulga, et al. The ReaxFF reactive force-field: development, applications and future directions. NPJ Computational Materials, 2:15011, 2016. 
[41] Georg Kresse and Jürgen Furthmüller. Efficient iterative schemes for ab initio total-energy calculations using a plane-wave basis set. Physical Review B, 54(16):11169, 1996.

[42] Georg Kresse and Jürgen Furthmüller. Efficiency of ab-initio total energy calculations for metals and semiconductors using a plane-wave basis set. Computational Materials Science, 6(1):15-50, 1996.

[43] John P Perdew, Kieron Burke, and Matthias Ernzerhof. Generalized gradient approximation made simple. Physical Review Letters, 77(18):3865, 1996.

[44] Max Dion, Henrik Rydberg, Elsebeth Schröder, David C Langreth, and Bengt I Lundqvist. Van der Waals density functional for general geometries. Physical Review Letters, 92(24):246401, 2004.

[45] Jiří Klimeš, David R Bowler, and Angelos Michaelides. Van der Waals density functionals applied to solids. Physical Review B, 83(19):195131, 2011.

[46] Graeme Henkelman, Blas P Uberuaga, and Hannes Jónsson. A climbing image nudged elastic band method for finding saddle points and minimum energy paths. The Journal of Chemical Physics, 113(22):9901-9904, 2000.

[47] Scott Kirkpatrick. Optimization by simulated annealing: Quantitative studies. Journal of Statistical Physics, 34(5-6):975-986, 1984.

[48] Steve Plimpton. Fast parallel algorithms for short-range molecular dynamics. Journal of Computational Physics, 117(1):1-19, 1995.

[49] Alejandro Strachan, Tahir Çağin, and William A Goddard III. Phase diagram of MgO from densityfunctional theory and molecular-dynamics simulations. Physical Review B, 60(22):15084, 1999.

[50] Alejandro Strachan, Tahir Çă̆in, Ŏ̆uz Gülseren, Sonali Mukherjee, Ronald E Cohen, and William A GoddardIII. First principles force field for metallic tantalum. Modelling and Simulation in Materials Science and Engineering, 12(4):S445, 2004.

[51] Graeme Henkelman, Andri Arnaldsson, and Hannes Jónsson. A fast and robust algorithm for Bader decomposition of charge density. Computational Materials Science, 36(3):354-360, 2006.

[52] Xiaofeng Qian, Junwei Liu, Liang Fu, and Ju Li. Quantum spin hall effect in two-dimensional transition metal dichalcogenides. Science, 346(6215):1344-1347, 2014.

[53] Dong Hoon Keum, Suyeon Cho, Jung Ho Kim, Duk-Hyun Choe, Ha-Jun Sung, Min Kan, Haeyong Kang, Jae-Yeol Hwang, Sung Wng Kim, Heejun Yang, et al. Bandgap opening in few-layered monoclinic MoTe2. Nature Physics, 11(6):482-486, 2015. 
[54] Tao Liang, Simon R Phillpot, and Susan B Sinnott. Parametrization of a reactive many-body potential for Mo-S systems. Physical Review B, 79(24):245110, 2009.

[55] Kimberly Chenoweth, Adri CT van Duin, and William A Goddard. The ReaxFF Monte Carlo reactive dynamics method for predicting atomistic structures of disordered ceramics: application to the Mo3VOx catalyst. Angewandte Chemie International Edition, 48(41):7630-7634, 2009.

[56] Adri CT van Duin, Vyacheslav S Bryantsev, Mamadou S Diallo, William A Goddard, Obaidur Rahaman, Douglas J Doren, David Raymand, and Kersti Hermansson. Development and validation of a ReaxFF reactive force field for $\mathrm{Cu}$ cation/water interactions and copper metal/metal oxide/metal hydroxide condensed phases. The Journal of Physical Chemistry A, 114(35):9507-9514, 2010.

[57] Hannu-Pekka Komsa and Arkady V Krasheninnikov. Electronic structures and optical properties of realistic transition metal dichalcogenide heterostructures from first principles. Physical Review B, 88(8):085318, 2013.

[58] R Soni, P Meuffels, A Petraru, M Weides, C Kügeler, R Waser, and H Kohlstedt. Probing cu doped Ge0.3Se0.7 based resistance switching memory devices with random telegraph noise. Journal of Applied Physics, 107(2):024517, 2010.

[59] David M Guzman, Nicolas Onofrio, and Alejandro Strachan. Stability and migration of small copper clusters in amorphous dielectrics. Journal of Applied Physics, 117(19):195702, 2015.

[60] Gan Ren, Rui Shi, and Yanting Wang. Structural, dynamic, and transport properties of concentrated aqueous sodium chloride solutions under an external static electric field. The Journal of Physical Chemistry B, 118(16):4404-4411, 2014. 